\title{
Stress, Food Addiction and Brain: Molecular Determinants and its Neural Networks Using Omics Platform
}

\author{
Hughes MR, Lee YJ, Heck DE and Kim HD* \\ Department of Public Health, School of Health Sciences and Practice, New York Medical College, USA
}

"Correspondence: Hong Duck Kim, Department of Public Health, School of Health Sciences and Practice, New York Medical College, USA

Received on 16 December 2019; Accepted on 23 January 2020; Published on 29 January 2020

Copyright (C) 2020 Hughes MR. This is an open access article and is distributed under the Creative Commons Attribution License, which permits unrestricted use, distribution, and reproduction in any medium, provided the original work is properly cited.

\begin{abstract}
Obesity is a complicated metabolic malfunction linked to disconnected signaling between interorgans, and inter-molecularly affected by impairment of the neuronal circuit, stemming from environmental stressors in children and adults. Upon environmental intoxication or change in the surrounding environmental stressors; the signaling for hunger could affect regulatory signaling in energy consumption and lead to a wide range of metabolic disruption and sensitivity, cell-to-cell and organ-to-organ. Presently, food addiction is associated with substance-based and behavioral malfunction, which reflects the alteration of molecular and genetic deficiency in animal studies and clinical studies. The prevailing food addiction hypothesis suggest that food types can alter the brain circuitry for a reward compensation which may link to compulsive eating behavior phenotype comparable to drug addiction. Like synthetic drugs, food consumption results in the release of dopamine, which relates to the feelings of being rewarded, and has shown to have similar brain activation patterns. It remains unclear what molecules could engage in the regulatory process, or what are key molecular determinants to control the hormonal secretion. Several interventions such as calorie restriction, exercise, bariatric surgery, and treatments for obesity management have been moderately successful in reducing body fat in the short term, but most fail to maintain long-term weight loss. Herein, we propose the advantage of the molecular-based risk assessment, namely the omics platform, to improve health quality by reviewing the impact of environmental stressors. Bulimia nervosa has received less attention in this regard, despite their regular binge eating symptoms.
\end{abstract}

Keywords: metabolic disorder, obesity, food addiction, neural circuit, cravings, omics

Abbreviations: BMI: body mass index; fMRI: Functional MRI; YFAS: Yale Food Addiction Scale 


\section{Introduction}

The rise in obesity rates worldwide can be attributed to overeating, sedentary lifestyle and even genetic predisposition. However, environmental and genetic factors that interact biologically with the body and neuroadaptation in response to eating habits in animal models have been found to be an underlying contribution to the pandemic or binge eating symptoms such as bulimia nervosa (BN) [1-3]. What causes food addiction? Is it the food in itself that is addictive? How much does stress contribute to the changes in eating behavior? There has been a call for further research into the link between eating behavior, food addiction, stress and the role they play in obesity risk factors with potential implications for treatment and prevention strategies [4]. Several research studies have expounded on possible pathways in the cause of stress related eating disorders and others explore the potential addictive quality of hyperpalatable food; though, some question the existence of food cravings or food addiction altogether [4, 5]. Hebebrand et al. examined the importance of properly understanding and characterizing addiction, through exploring the perspectives of clinicians and researchers [1]. Such characterizations of addiction are compulsive seeking behavior a) from loss of control in substance use, b) to alleviate negative emotional state, and c) engagement in behavior to fulfill effects of appetite. The National Institute on Drug Abuse defines addiction as "a chronic, relapsing brain disease that is characterized by compulsive drug seeking and use, despite harmful consequences." There are some difficulties in prescribing the measurements to these elements, as they may not be independent of each other, but operate in rather complex circuitries.

Another term seen in relation to food addiction is "craving". Craving is described as a strong desire for an item i.e., food or drugs and many parallels were reported between food and drug cravings which may result in deem food addiction [6]. Pérez-Ortiz et al. pointed out that the majority of clinical criteria for addiction is based on the negative outcome of continued substance use or the failure to discontinue. This can be difficult to apply to food since food consumption generally has positive effects. Although, Pérez-Ortiz et al. does point out that like drug addicts, some obese and overweight individuals meet the addiction criterion because they exhibit persistent desire (compulsive seeking behavior), repeated unsuccessful attempts to quit (preoccupation with behavior) and reduction in occupational, social and recreational activities (preoccupation with behavior, temporary satisfaction, loss of control and negative effects). A popular hypothesis is that food addiction occurs as a result of highly palatable food causing strong activation in the reward circuitry similar to addictive drugs [7]. Like synthetic drugs, food overconsumption results in the release of dopamine which relates to the feelings of reward and has shown to have similar brain activation patterns $[1,8]$. Parts of the brain are responsible for driving motivation for food reward and are activated by certain environmental cues, such as the orbitofrontal cortex (OFC), amygdala, insula, striatum, anterior cingulate cortex (ACC) and the dorsolateral prefrontal cortex (DLPFC) [1, 2, 8]. These systems regulate all aspects of feeding behavior which are closely interlinked, such as eating for caloric and nutritional need as well as eating for pleasure and satisfaction [1].

\section{Food, stress and craving: quantitative analysis with imaging tool}

Craving (a strong desire for a particular food) is a neural process between limbic and striatal to adapt stress response in the brain, which has been associated with body mass index (BMI) due to skewed pattern of food types including high sugar or high fat food which leads to dietary restriction failure followed by binge eating and addictive eating [6, 9]. Functional MRI (fMRI) and positron emission tomography (PET) show that during a craving, there is a strong activation of the sensory memory-related area, i.e., the striatum, prefrontal cortex, the insula and anterior cingulate cortex caudate, which are associated with dopamine neurons of the ventral tegmental area in drug craving [10]. Compulsive eating behavior can develop through the consumption of highly palatable food in response to uncontrollable stress that can trigger neurological adaptation. They define potential stress as a process involving perception, appraisal and response to noxious events or stimuli [5]. Stress can be emotional (termed ego-threatening) and physical $[4,5]$. In exploring how extrinsic factors play a role in eating habits, Scott et al. suggest that $80 \%$ of people change their eating pattern when they perceive themselves to be stressed by either overeating or undereating, and that chronic and acute stress impact eating behaviors. This effect is more pronounced in obese individuals with 
increased instances of stress-related binge eating [4]. They found an association between high-stress levels and an increase in fat consumption. Similarly, they suggest that food with high fat and/or high sugar content is the food of choice during highly stressful stimuli [5]. This was demonstrated in an animal study where rats, when presented with a choice of a highly palatable food, will increase consumption of that food source more consistently exposed to stress. In a previous study to human, the similar counteract effects of stress in both palatable foods (i.e., fast food and snacks) and other calorie-dense (known as comfort food) was reported [5, 11]. A similar mouse model was developed by Pérez-Ortiz et al. where they explored the term addiction and how highly palatable food and stress play a role in addictive behavior focused on the effect of neurological and physiological changes on food addiction in response to food consumption and stressors using animal model. The result indicates that high fat diet coupled with periods of food deprivation increased biomarkers for addiction. A study indicated that nutritional deprivation was necessary to produce food cravings by placing subjects on a nutritionally adequate but restrictive and boring diet for 5 days. There was an increase in frequency of food cravings in the 5-day phase using isolate brain activation. In the experiment design, one group was treated with nutritionally adequate but boring, restrictive diet and the other group was treated on an unrestricted diet including the boring diet. All subjects were asked to imagine foods they really liked and compared the response of those group after scanning. There were three different representative studies reported that utilized Yale Food Addiction Scale (YFAS) measurable tool and/or meta-analysis. Gearhardt et al. conducted the hypothesis if high food addiction scores were associated with similar neural activation patterns as substance dependence. fMRI was adopted in the study of YFAS (Likert Scale version) to measure response receipt and anticipated receipt to highly palatable food. Another study lead by Pursey et al. indicated that the pattern of food addiction followed by the application of YFAS scale is related to age-dependent, sex, and the metabolic symptomsensitive trend [12]. Other studies investigated the correlation between food addiction and BMI using YFAS 2.0 tool. The outcome of the study suggests that food addiction could be linked to eating behavioral patterns like overeating in underweight populations [13]. In addition, it was reported on the correlation between the frequency of food craving and eating behavioral patterns in young populations.

In brief, total of 48 women with an average age of 20.8 and BMI ranging from 23.8-39.2 were screened for the study with exclusion of individuals with pre-identified binge eating behaviors, illicit drug use or psychotropic medication use, smokers, head injury with loss of memory and current diagnosis. 39 women as final sample size were used due to eliminations from missing data from YFAS and excessive head movement during MRI. Participants received a baseline scan and were asked to eat regular meals but refrain from consuming caffeinated beverages for 4-6 h before scan to capture hunger state that would occur before next meal. Chocolate milkshake was used as the stimuli to examine brain activation in response to anticipated consumption and consumption of highly palatable food and a calorie free tasteless solution was prepared (to mimic the taste of saliva). The findings indicate that there was a large increase in food craving frequency reported during the 5-day restricted diet, demonstrating that nutritional deficiency is not necessary to produce food craving $[8,14,15]$.

Similarly, another group investigated the localization of the brain activation site, which could be involved in food craving. They found that all participants on the diet had exhibited food cravings and craving-specific activation was observed on MRI imagining that involved the nucleus, hippocampus and insula. Furthermore, sensory memory was found to play a central role in the experience of food craving and may be a learned behavior acquired by repeatedly eating craved food when hungry. These results indicate that an addict has an urge to engage in their behavioral routine, and any changes to that routine can cause discomfort resulting in cravings and/or withdrawal symptoms. However, it is the routine of eating that is addictive and not the substance that make up the food. [1] It is necessary to support the premise by further stating the link between hunger, satiation and satiety with the reward system is the foundation of addictive-like eating behavior. In other study conducted by Gearhardt et al., it was found that obese individuals show greater activation in regions of the brain that is coded for reward stimuli and similar to drug addiction, greater activation in regions associated with drug related cravings. Although, in hypersensitivity, it was also noted that there is a reduced reward circuitry stimulus resulting in weaker subjective reward, which lead individuals to overeat due to experiencing less reward from eating [14]. This is also similarly seen in drug addiction leading to drug abuse. There 
was also an association between high food addiction scores and high activation in the orbitofrontal cortex and anterior cingulate cortex, regions that play a role in food cues and the motivation to eat (as well as consume drugs if there is substance dependence). The higher the food addictions score, the stronger the motivation to seek out food in response to food related cues. Although, food consumption is known to activate these reward systems, it suggests that this activation does not necessarily implicate specific food substances as addictive, but that the act of eating is what initiates the addictive behavior [1].

\section{Food addiction, regulatory sensor and visualization in the brain}

There is a growing body of evidence in animal and human studies which strongly demonstrates neurological changes which occurs in the brain under chronic stressors that are similar to drug addiction when highly palatable foods are consumed. The phenomenon of food addiction is being widely explored due to the rising obesity epidemic that goes beyond just overindulgence of high calorie food, sedentary lifestyle, gender, and even genetic predisposition to fat storage $[11,14,15]$. Addictive behavior lies at the core of why so many people fail to maintain weight loss or stick to restrictive food regiments to maintain a healthy weight. Hebebrand et al. prefers the term "eating addiction" instead of "food addiction," because of the implied nature of a substance-related phenomenon [1]. As previously mentioned, they assert that it is the act of eating that imitates the addictive eating behavior. Although, high-fat and high-sugar food itself do trigger the reward center in the brain, repeated exposures do create stronger motivation to choose such food. However, studies fail to point any specific ingredient in food that is the culprit of the addictive behavior. The research shows a reciprocal relationship between stress and addictive behaviors, not exactly how food addiction plays a role. Although, comfort food is used to cope with stress, Leigh et al. point out that compulsive eating in this regard may be a result of disordered coping mechanism and not addictive food properties. Especially, since food is a necessary part of life and the act of eating evokes positive feelings that promote an essential life preserving behavior. [8] Medications used to treat drug abuse are also being considered as potential interventions for weight loss. According to Sinha et al., some evidence suggest that weight maintenance is associated with low stress levels and the ability to cope with stress. Since, stress promotes food cravings and binge eating, it is useful to reduce stress in weight management programs [4].

The need to eat is programed into the brain to ensure energy intake and calorie hemostasis [14]. Energy consumption is essential for the survival of all living creatures, but humans no longer simply eat to survive. Food addiction combines concepts of substance based and behavioral addiction which has been consistent with human and animal study data $[1,8]$. The abundance of highly palatable, low nutrition and inexpensive food has resulted in non-homeostatic eating habits. The prevailing food addiction hypothesis is that these types of food alters the brain circuitry for reward compelling a compulsive eating behavior phenotype comparable to drug addiction [7]. Like drugs, food consumption results in the release of dopamine which relates to the feelings of reward and has shown to have similar brain activation patterns. [8] In exploring how extrinsic factors play a role in eating habits, it was reported that $80 \%$ of people change their eating pattern when they perceive themselves to be stressed by either overeating or undereating and that chronic and acute stress impact eating behaviors [4]. This effect is more pronounced in obese individuals with increased instances of stress-related binge eating [10]. Several interventions and treatments for obesity have been moderately successful in reducing body fat in the short-term but most fail to maintain long-term weight loss. They have one thing in common and that is they do not address the underlying dysfunctionality that lead to excessive eating behavior [7].

\section{Conclusion and Future Perspective}

Even though progress has been made in the concept of hunger, stress, craving network; it still remains unclear how to define determinants of molecules which could interact with the sensitive or resistant gene expressions under cravings signaling, such as the initiation, propagation and termination of receptors with soluble mediators or the neurotransmitter-dependent manner of receptors. To make advancements in understanding the molecular landscape of craving signaling on the metabolic disorder, we need to manipulate gene-phenotype specific relations after defining what environmental stressors cause or activate the eating signals. Identifying the risk underlying social determinants 
which eating signals trigger, the presence or absence of behavioral change in a phenotype-dependent manner using proteomics (Omic-derived platform) in conjunction with risk factors (i.e., genetic and environmental cues). Validation of the risk could be framed using metabolomics which can be examined in the animal model or utilize translational pre-clinic samples [2]. To further visualize the regulatory signaling and phenotypic alteration as part of functional aspects in food addiction, one can utilize molecular-driven gene targeting along with functional analysis armed with the omics platform. Future direction may help to understand the cause of food behavioral change utilizing the systemic approach, such as metabolomics, nutrigenomics, neuro-genetics, and epigenetic visualization to unveil the alternation of molecular pattern in food addiction and overeating behavioral trait.

\section{Conflicts of Interest}

The authors have no conflict of interest to declare.

\section{References}

1. Hebebrand J, Albayrak Ö, Adan R, et al. "Eating addiction", rather than "food addiction", better captures addictive-like eating behavior. Neurosci Biobehav Rev. 2014;47:295-306.

2. Novelle MG, Diéguez C. Food addiction and binge eating: lessons learned from animal models. Nutrients. 2018;10(1):E71.

3. Meule A, von Rezori V, Blechert J. Food addiction and bulimia nervosa. Eur Eat Disord Rev. 2014;22(5):331-37.

4. Scott C, Johnstone AM. Stress and eating behaviour: implications for obesity. Obes Facts. 2012;5(2):277-87.

5. Yau YH, Potenza MN. Stress and eating behaviors. Minerva Endocrinol. 2013;38(3):255-67.

6. Sinha R. Role of addiction and stress neurobiology on food intake and obesity. Biol Psychol. 2018;131:5-13.

7. Pérez-Ortiz JM, Galiana-Simal A, Salas E, et al. A high-fat diet combined with food deprivation increases food seeking and the expression of candidate biomarkers of addiction. Addict Biol. 2017;22(4):1002-09.

8. Leigh SJ, Morris MJ. The role of reward circuitry and food addiction in the obesity epidemic: An update. Biol Psychol. 2018;131:31-42.

9. Volkow ND, Wang GJ, Tomasi D, et al. Obesity and addiction: neurobiological overlaps. Obes Rev. 2013;14(1):2-18.

10. Tanabe J, Regner M, Sakai J, et al. Neuroimaging reward, craving, learning, and cognitive control in substance use disorders: review and implications for treatment. Br J Radiol. 2019;92(1101):20180942.

11. Sinha R, Jastreboff AM. Stress as a common risk factor for obesity and addiction. Biol Psychiatry. 2013;73(9):827-35.

12. Pursey KM, Stanwell P, Gearhardt AN, et al. The prevalence of food addiction as assessed by the Yale food addiction scale: a systematic review. Nutrients. 2014;6(10):4552-90. 
13. Hauck C, Weiß A, Schulte EM, et al. Prevalence of 'Food Addiction' as measured with the Yale food addiction scale 2.0 in a representative german sample and its association with sex, age and weight categories. Obes Facts. 10(1):12-24.

14. Gearhardt AN, Yokum S, Orr PT, et al. Neural correlates of food addiction. Arch Gen Psychiatry. 2011;68(8):808-16.

15. Gold MS, Badgaiyan RD, Blum K. A shared molecular and genetic basis for food and drug addiction: overcoming hypodopaminergic trait/state by incorporating dopamine agonistic therapy in psychiatry. Psychiatr Clin North Am. 2015;38(3):419-62. 